\title{
APPLYING AI IN TIMES OF COVID-19
}

\author{
Vishnu Singh Sengar, Snigdha Das \\ Department of CSE \& IT \\ Jaypee Institute of Information Technology, Noida, Uttar Pradesh, India
}

\begin{abstract}
This study is aimed to provide efficient solutions to ease the workload on humans and also to lessen the human interaction. As advised a distance of $6 \mathrm{ft}$ is considered safe in COVID times so this study aims to monitor the distances between individuals through the public security CCTV cameras using techniques of Computer Vision and Deep learning.
\end{abstract}

Monitoring distances with a single camera is still an ongoing research and still with the use of basic math and coordinates knowledge we could find out the approximate distances between individuals. We can make detections by using TensorFlow object detection API and calculate distance by looking between the separation of coordinates of their centroids.

These types of solutions will enable to monitor public gathering using existing architecture. Good wide angle camera will enable to keep an eye better than humans can do. This will help lessen the deployment of police officials in these places enabling more effective use of our police force.

Keywords-Computer Vision, TensorFlow, Social Distancing, Deep learning.

\section{INTRODUCTION}

COVID-19 as we all know is very contagious disease which is spreading rapidly throughout the world by human contact. A drop of cough or the tiny molecules of our saliva which falls out while talking may also lead to transmission of the COVID virus from an infected person to others. The main symptoms which come with this disease are flu like including cough, fever and shortness of breath [1]. To stop this a guideline is issued by responsible authorities maintain a distance of at least $6 \mathrm{ft}$ from other people around. This is what we call, Social Distancing [2]. It's not always possible to position the police officials to keep a check on social gathering and not feasible as well. To help solve this problem, AI which would works faster and also generates accurate results can be relied on.

Monitoring is done over existing architecture with the preinstalled CCTV cameras at various public places using OpenCV [3]. This helps not only managing social crowd but also help manage industrial workers where it's hard for humans to look into every corner of factories. Cameras on the other hand are present in most of the sections and thus monitor in a more efficient way.

To detect out the persons many pre-trained models like SSD MobileNet [4] are available which produce good results in shorter period of time. They are trained over COCO Dataset [5] which consists of millions of images belonging to several categories which further boosts model accuracy.

\section{LITERATURE SURVEY}

In the recent times with the growing integration of $\mathrm{AI}$ in our day to day lives, Computer Vision has become a vital inevitable branch which is playing an important role in automation. Be that self-driving cars where cameras are placed on the dashboard and with techniques like Inverse Perspective Mapping, a bird eye view is generated in real time which helps in calculating the distances between a car and other objects around [6].

Complex systems are also coming up which uses a rotating mirror and the property that far away images will have a less pixel change when mirror is rotated rather than images closer to the camera [7]. Such systems are of great importance when we have limited resources like having a fixed normal RGB camera instead of complex architectures which consists of multiple cameras to enhance 3D view. Modifying a camera so as to make its image sensor inclined at certain angle, will make the image defocused at different areas. The most focussed image is at the projection plane. Finding the distance between lens and projection plane will give us the actual distance of image [8].

Some similar advanced methods like we used, propose targeting of a laser over the object we want to calculate distance of. Using the fact that objects at different distances will have different diameters of projected laser a relationship is made between its distance and the diameter [9].

\section{PROPOSED ALGORITHM}

To implement the Idea of keeping a check on distances among individuals, we made use of basics of Coordinate Geometry. We used ssd_mobilenet_v1_coco_2018_01_28 to detect the objects in the images and then took out the coordinates of the bounding box if it encloses a person. Then we calculated the centroid of the box by using:

$$
\begin{aligned}
& \mathrm{X}_{\mathrm{c}}=\left(\mathrm{X}_{1}+\mathrm{X}_{2}\right) / 2 \\
& \mathrm{Y}_{\mathrm{c}}=\left(\mathrm{Y}_{1}+\mathrm{Y}_{2}\right) / 2
\end{aligned}
$$


Where

$X_{c}, Y_{c}=X$ and $Y$ coordinates of centroid respectively.

$\mathrm{X}_{1}, \mathrm{X}_{2}=\mathrm{X}$-Coordinates of top left and bottom right point of bounding box

$\mathrm{Y}_{1}, \mathrm{Y}_{2}=\mathrm{Y}$-Coordinates of top left and bottom right point of bounding box

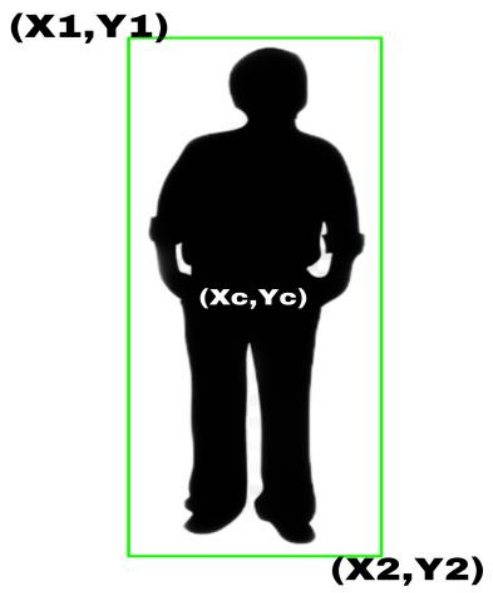

Figure 1 Calculation of Centroid

All the centroids detected are then saved to a dictionary. Now we have to find the distances between all of the centroid pairs. We calculated the distance using the formula:

$$
D=\sqrt{(X 1-X 2)^{2}+(Y 1-Y 2)^{2}}
$$

Where,

$\mathrm{X}_{\mathrm{i}}=\mathrm{X}$ coordinates of point $\mathrm{i}(\mathrm{i}=1,2)$

$\mathrm{Y}_{\mathrm{i}}=\mathrm{Y}$ coordinates of point $\mathrm{i}(\mathrm{i}=1,2)$

We then define a safe distance between two centroids which will account to $6 \mathrm{ft}$ and that by experiments is found to be 75 . Thus if the distance between centroids of two persons is less than or equal to 75 then they are not following social distancing and we displayed a red bounding box along the persons of that pair by looking at the person ID which we uniquely assign to each of the detected humans in the frame. We also flashed Social Distancing Void warning on the output screen so that it becomes visually easier for the person monitoring hundreds of such output screens from hundreds of camera feeds. If the distance comes out to be greater than 75 then we displayed a green bounding box along that pair of persons.

Shown here is a graphical representation of above told process.

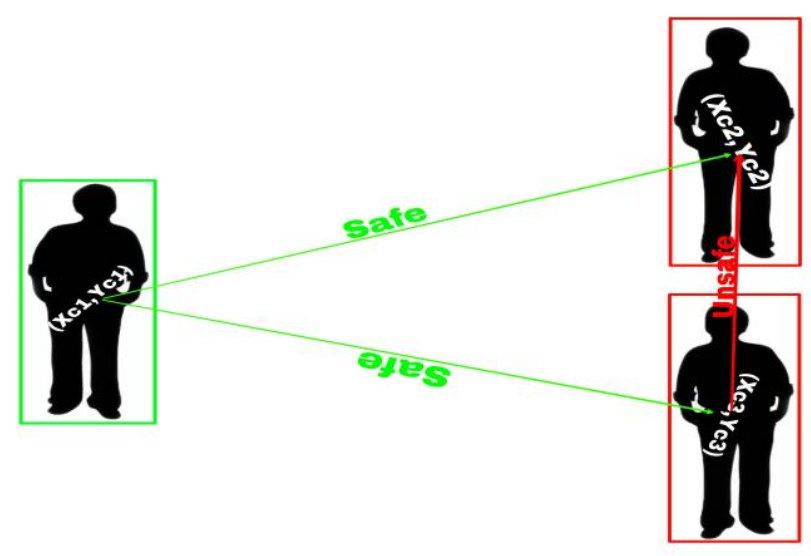

Figure 2 Calculations of Distances

In general, we followed these steps.

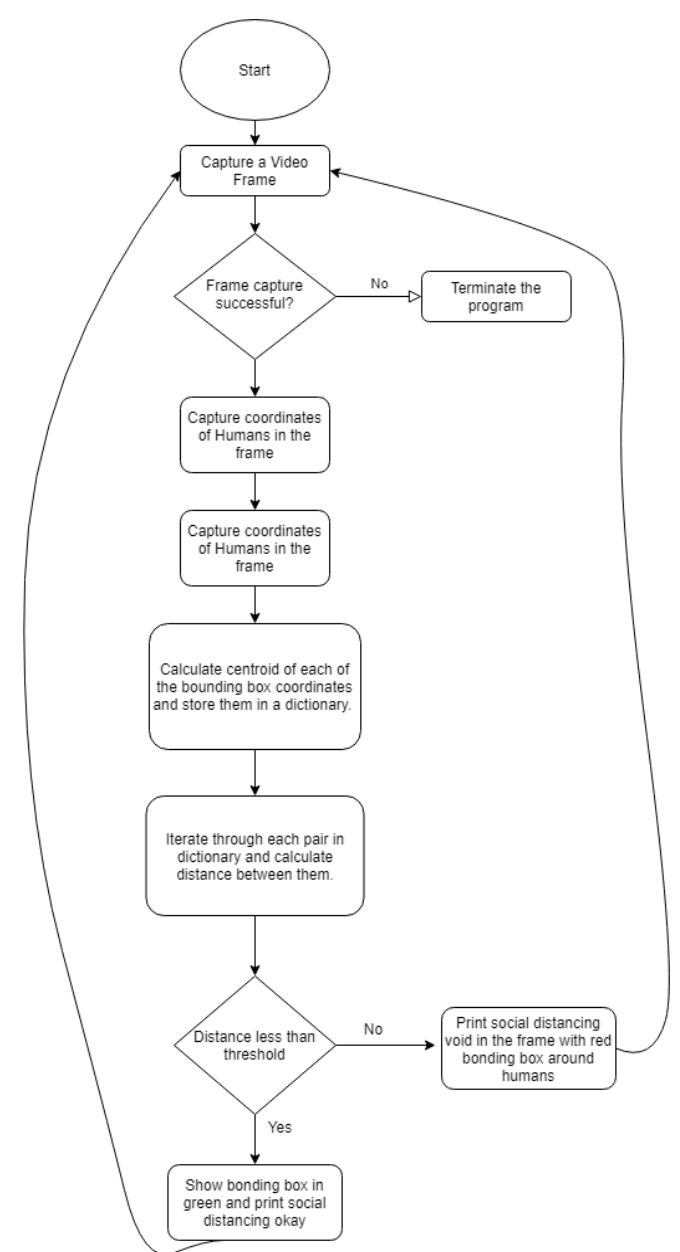

Figure 3 Flow Chart of The Given Algorithm

\section{EXPERIMENT AND RESULTS}

After applying the above algorithm, we obtained good results. We scrapped some videos on the internet and tried to run our 


\section{International Journal of Engineering Applied Sciences and Technology, 2020 \\ Vol. 5, Issue 1, ISSN No. 2455-2143, Pages 314-318 \\ Published Online May 2020 in IJEAST (http://www.ijeast.com)}

model on them and it worked quite well. We tested our model on Social Distancing(COVID-19) dataset from Kaggle [10] which contains a video file in which number of people are passing in the streets of Oxford University. Below is the output of the same.

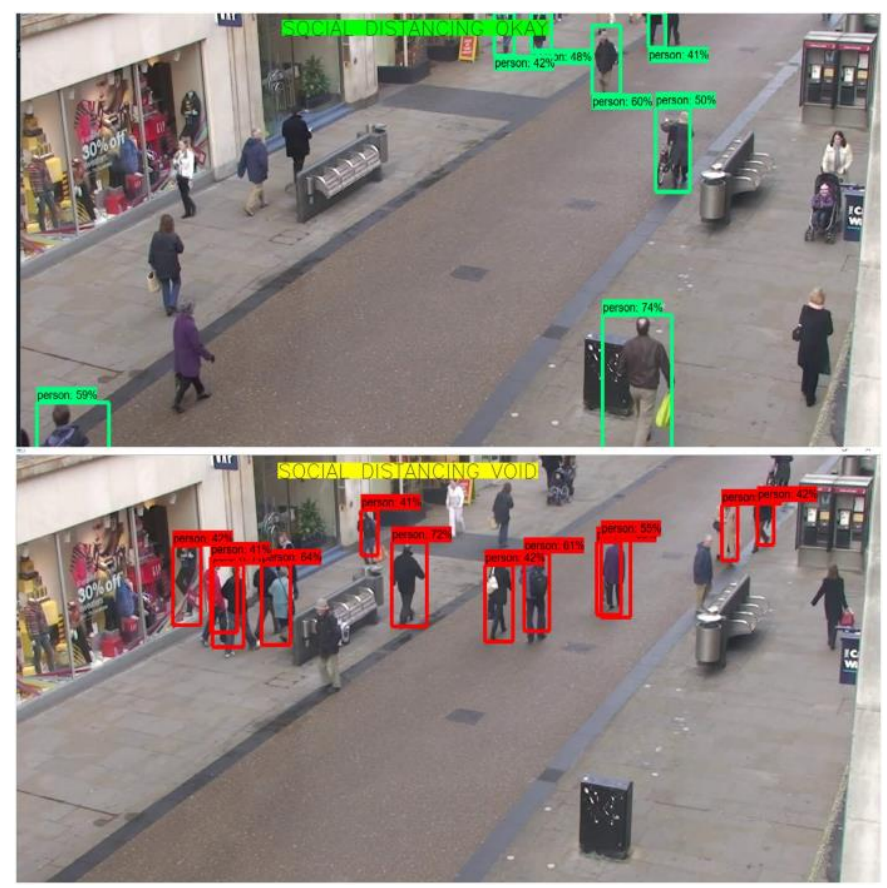

Figure 4 People walking on the streets at various time intervals

As you can see in figure 4, in the top picture frame people are at a good distance and hence the model displayed Social Distancing Okay and all the persons are having green boxes. While the bottom frame has congestion of people and hence people violating are shown in red boxes and model flashed Social Distancing Void at the top of screen.

We tested our model on the famous game of Australian Open Final in 2012 between Rafael Nadal and Novak Djokovic and we got good results. You can see in Figure 5 in the start of the game when players shook hands then since they were close they violated social distancing and hence model turned their detection boxes red and flashed Social Distancing Void on display.

In figure 6 on the other hand the game was started and since the players were at distance greater then $6 \mathrm{ft}$ our model showed them in green bounding boxes and displayed Social Distancing Okay on the screen.
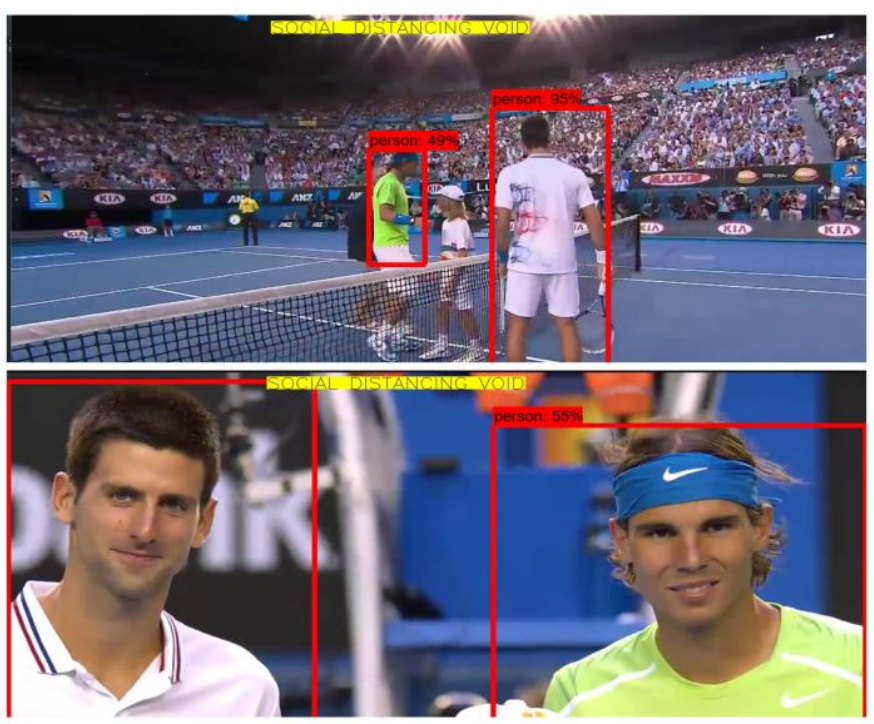

Figure 5 The opening ceremony of Australian Open Finals - 2012 (SRC-YouTube)
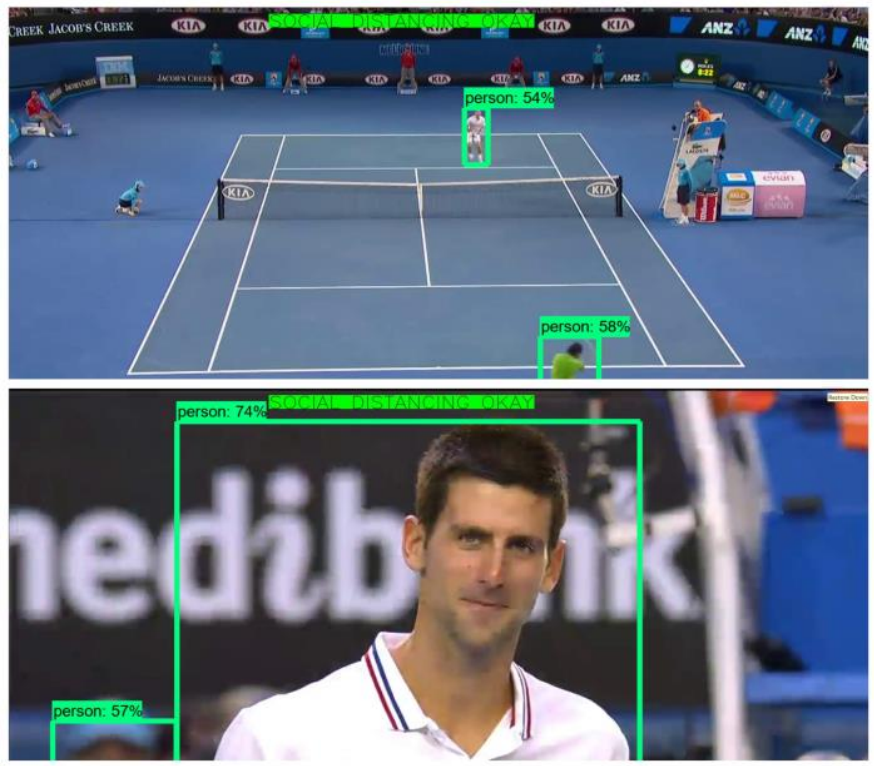
Figure 6 Ongoing Game of Australian Open Finals - 2012

We tested our model on ourselves (fig 7,8) as well. In Figure 6 we were at safe distances from each other hence the model displayed Social Distancing Okay on the screen but when the distance decreased below that permissible (figure 8), model changed its decision and displayed Social Distancing Void in the screen window. 


\section{International Journal of Engineering Applied Sciences and Technology, 2020 \\ Vol. 5, Issue 1, ISSN No. 2455-2143, Pages 314-318 \\ Published Online May 2020 in IJEAST (http://www.ijeast.com)}
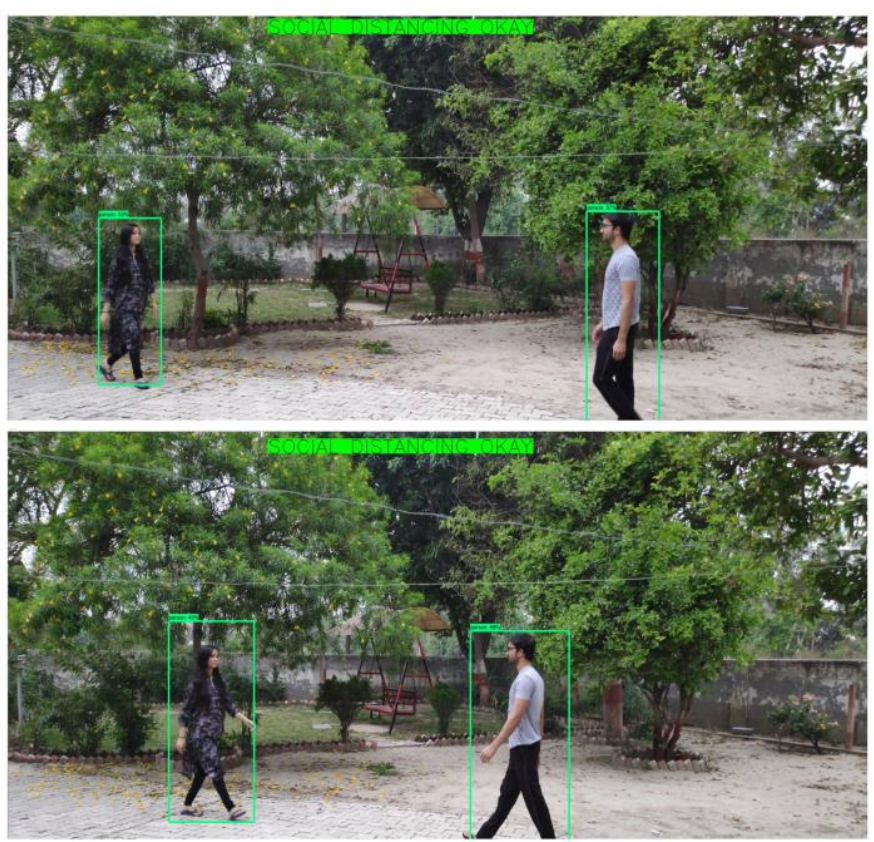

Figure 7 Distance greater then permissible value

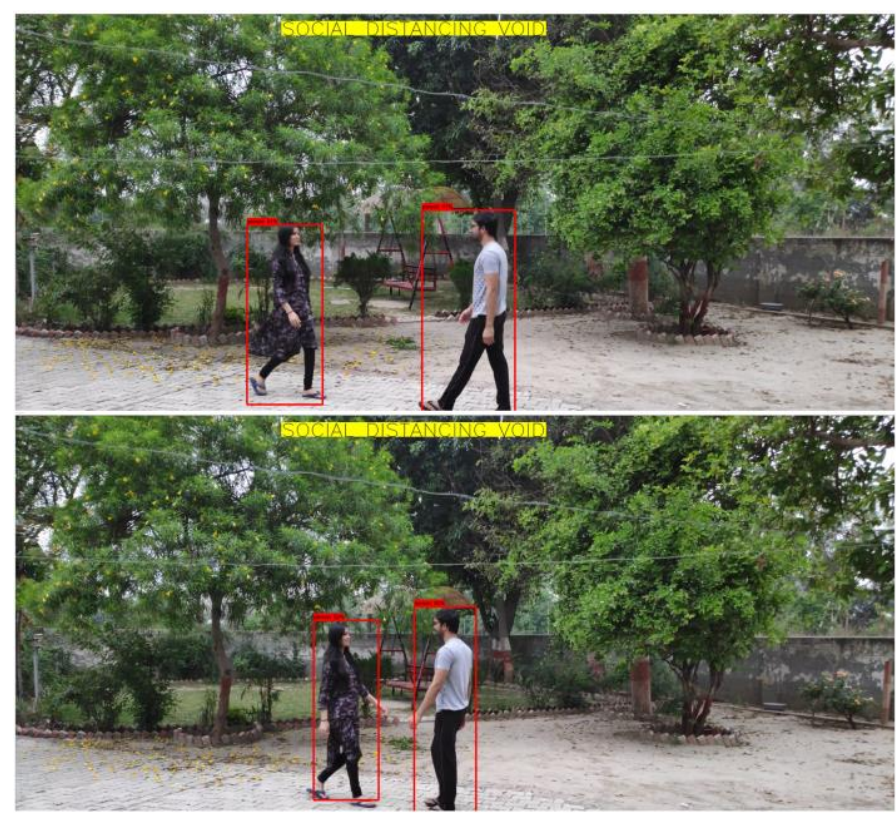

Figure 8 Distance decreased below permissible value

\section{FUTURE SCOPE}

Distance measurement using a single RGB camera is not always accurate. We must come up with an infrastructure with depth sensing cameras which calculates depth between two persons more accurately.
Deployment of speakers in public places which could be used to issue warnings to the crowd when they disobey social distancing norms could allow remote management of crowd.

Processing image frames in some other form like treating the instances of persons as points could help reduce the time for processing and hence will in-turn result in even faster systems.

\section{CONCLUSION}

This social distancing model gave accurate results and thus came out to be highly trustworthy. Seeing the rising concerns around the globe for such pandemic, the deployment of such AI technologies must be encouraged. This will help make our public places safe and also facilitate better deployment of our police officials. When used inside factories and other industries, it'll help their healthy working which in-turn will boost country's economic growth.

\section{REFERENCES}

[1] Xu, Z., Shi, L., Wang, Y., Zhang, J., Huang, L., Zhang, C., Liu, S., Zhao, P., Liu, H., Zhu, L. and Tai, Y., 2020. Pathological findings of COVID-19 associated with acute respiratory distress syndrome. The Lancet respiratory medicine, 8(4), pp.420-422.

[2] Reluga, T.C., 2010. Game theory of social distancing in response to an epidemic. PLoS computational biology, 6(5).

[3] Bradski, G., 2000. The OpenCV Library. Dr. Dobb\&\#39; sournal of Software Tools.

[4] Davis Andy,Dean Jeffry, Devin Matthieu, Ghemawat Sanjay, Goodfellow Ian, Harp Andrew, Irving Geoffrey, Isard Michael, Jozefowicz Rafal, Jia Yangqing, Kaiser Lukasz, Kudlur Manjunath, Levenberg Josh, Mané Dan, Schuster Mike, Monga Rajat, Moore Sherry, Murray Derek, Olah Chris, Shlens Jonathon, Steiner Benoit, Sutskever Ilya, Talwar Kunal, Tucker Paul, Vanhoucke Vincent, Vasudevan Vijay, Viégas Fernanda, Vinyals Oriol, Warden Pete, Wattenberg Martin, Wicke Martin, Yu Yuan, and Zheng Xiaoqiang (2015) TensorFlow: Large-scale machine learning on heterogeneous systems. Software available from tensorflow.org.

[5] Lin TY. et al. (2014) Microsoft COCO: Common Objects in Context. In: Fleet D., Pajdla T., Schiele B., Tuytelaars T. (eds) Computer Vision - ECCV 2014. ECCV 2014. Lecture Notes in Computer Science, vol 8693. Springer, Cham

[6] Tuohy, Shane, et al. "Distance determination for an automobile environment using inverse perspective mapping in OpenCV." (2010): 100-105.D. Kunder, "Multi-resolution Digital Watermarking Algorithms and Implications for Multimedia Signals", Ph.D. thesis, university of Toronto, Canada, 2001. 
[7] Kim, Hyongsuk, et al.(2005) "Distance measurement using a single camera with a rotating mirror." International Journal of Control, Automation, and Systems 3.4: 542-551.

[8] L. Xiaoming, Qin Tian, Chen Wanchun and Y. Xingliang, "Real-time distance measurement using a modified camera," 2010 IEEE Sensors Applications Symposium (SAS), Limerick, 2010, pp. 54-58, doi: 10.1109/SAS.2010.5439423.

[9] Wang, T.H., Lu, M.C., Wang, W.Y., Tsai, C.Y., Rd, T.K. and County, T., 2007, August. Distance measurement using single non-metric CCD camera. In Proc. 7th WSEAS Int. Conf. Signal Process. Comput. Geom. Artif. Vision, Athens, Greece, Athens, Greece (pp. 1-6).

[10] Raj Pritesh. (2020, May). Social Distancing(COVID-19), Version 1. Retrieved May 3, 2020 from https://www.kaggle.com/priteshraj10/detect-socialdistancingcovid19 